\title{
Aktivitätskoeffizienten bei Elektrolytschmelzen mit mehreren Komponenten
}

\author{
JOACHIM RichteR
}

Institut für Physikalische Chemie der Rheinisch-Westfälischen Technischen Hochschule Aachen

(Z. Naturforsch. 24 a, 447-456 [1969]; eingegangen am 7. Dezember 1968)

Es wird eine Definition der Aktivitätskoeffizienten für binäre Elektrolytschmelzen aufgegriffen und so fortgeführt, daß man eine einzige Formel erhält, aus der man die Aktivitätskoeffizienten jeder beliebig zusammengesetzten Elektrolytschmelze ableiten kann. Für die Auswertung von EMKMessungen findet man mit dieser Formel für den Logarithmus des Aktivitätskoeffizienten $f_{k}$ der Komponente $k$ :

$$
\ln f_{k}=\frac{F\left(\Phi-\Phi_{0}\right)}{q R T}-\ln \frac{v_{k} v_{k} \prod_{k \mathrm{a}}\left(x_{k}+\sum_{l=1}^{p}\left(v_{l k} / v_{k \alpha}\right) x_{l}\right)^{v_{k \alpha}}}{\left(\sum_{i=1}^{n} v_{i} x_{i}\right)^{v_{k}}},
$$

worin $\Phi$ die EMK der isotherm-isobaren reversiblen galvanischen Kette mit den betrachteten Komponenten und $\Phi_{0}$ die EMK der Kette mit der reinen Komponente $k$ ist. $q$ ergibt sich aus der in der Kette ablaufenden Reaktion; $F$ ist die Faraday-, $R$ die Gaskonstante, $T$ die absolute Temperatur. Die $v$ und $x$ sind Zerfallszahlen und stöchiometrische Molenbrüche.

Es werden an Hand dieser Gleichung aus der Literatur experimentelle Werte der Aktivitätskoeffizienten ermittelt. Die bisher übliche Definition von anderen Aktivitätskoeffizienten wird kritisch untersucht.

In einer früheren Arbeit ${ }^{1}$ wurden die Grundlagen der thermodynamischen Beschreibung von binären Elektrolytschmelzen dargelegt. Dabei wurde auf allgemeine von Modellvorstellungen, wie der von TEM$\mathrm{KIN}^{2}$, unabhängige Weise der Typ einer „idealen Elektrolytschmelze“ eingeführt. Außerdem wurden zweckmäßige Aktivitätskoeffizienten definiert, die beim Grenzübergang zu den reinen flüssigen Komponenten immer Eins werden und die die Abweichung von der vollständig dissoziierten idealen Elektrolytschmelze beschreiben. Die Logarithmen der Aktivitätskoeffizienten lassen sich generell ${ }^{3}$ als Potenzreihen im Molenbruch darstellen. Die Notwendigkeit der Neufestlegung von Aktivitätskoeffizienten ist bereits an anderer Stelle ${ }^{4}$ ausführlich begründet worden.

In der vorliegenden Arbeit wird der Versuch gemacht, unter Zugrundelegung der von $\mathrm{HAASE}^{1}$ eingeführten Definitionen einen Ausdruck zu entwikkeln, mit dessen Hilfe man die Aktivitätskoeffizienten jeder beliebig zusammengesetzten Elektrolytschmelze explizit angeben kann. Dieser Ausdruck wird der Reihe nach für zwei-, drei- und vierkomponentige Systeme aufgestellt und so verallgemeinert, daß er uneingeschränkt gilt. Die entwickelten Gleichungen

1 R. HAase, Z. Phys. Chem. Frankfurt 63, 95 [1969].

2 M. Temkin, Acta Physicochim. URSS 20, 411 [1945]. werden mit Literaturdaten hinsichtlich ihrer Zweckmäßigkeit untersucht. Dabei stellt sich heraus, daß bestimmte Systemtypen binärer Elektrolytschmelzen in großer Zahl vermessen worden sind, während für andere kaum Messungen vorliegen. Auch ein Beispiel für ein ternäres System wird ausgewertet. Für ein System mit vier Komponenten konnte in der Literatur kein Beispiel nachgewiesen werden.

\section{Definition der Aktivitätskoeffizienten}

Nach HAase ${ }^{1}$ lautet die Definition der Aktivitätskoeffizienten in binären Elektrolytschmelzen

$$
\ln f_{j}=\psi_{j}-\psi_{j}^{\text {id }} \text {. }
$$

Der Aktivitätskoeffizient $f_{j}$ der Komponente $j$ ist dimensionslos und hängt wie üblich von der Temperatur $T$, dem Druck $P$ und dem stöchiometrischen Molenbruch $x_{j}$ ab. $\psi_{j}$ ist die Abkürzung für

$$
\psi_{j} \equiv\left(\mu_{j}-\mu_{0 j}\right) / R T \text {. }
$$

$\psi_{j}{ }^{\text {id }}$ ist der sinngemäße Ausdruck für die vollständig dissoziierte ideale Elektrolytschmelze. In Gl. (2) ist $\mu_{j}$ das chemische Potential der Komponente $j$ in der Mischung, $\mu_{0 j}$ das der reinen Komponente $j$ und $R$ die Gaskonstante.

3 Siehe: R. HaAse, Thermodynamik der Mischphasen, Verlag Springer, Berlin - Göttingen - Heidelberg 1956.

4 J. Richter, Ber. Bunsenges. Phys. Chem. 72, 681 [1968]. 
Wir behalten die Definitionen (1) und (2) für beliebig viele Komponenten bei, wobei $\psi_{j}^{\text {id }}$ später näher erläutert wird. Dann läßt sich zeigen, daß für die EMK $(\Phi)$ einer isotherm-isobaren reversiblen galvanischen Kette gilt:

$$
\ln f_{k}=\frac{F\left(\Phi-\Phi_{0}\right)}{q R T}-\psi_{k}^{\text {id }} .
$$

$F$ bedeutet die Faraday-Konstante und $\Phi_{0}$ die EMK der Kette mit der reinen Komponente $k . q$ ist der Faktor, der vor dem chemischen Potential der Komponente $k$ steht, wenn man die Affinität der zu $k$ gehörenden Heterogenreaktion hinschreibt. Daraus geht hervor, daß die Komponente $k$ immer diejenige Komponente des Systems ist, für deren Ionen die Elektroden der Kette reversibel sind.

Wir verwenden im weiteren folgende Indizierung: $i$ ist der Summationsindex für alle $n$ Komponenten des Systems $(i=1$ bis $n), j$ bezeichnet eine beliebige Komponente der $n$ möglichen, $k$ ist die Komponente, für deren Ionen die Elektroden der Kette reversibel sind und der Index $l$ bezeichnet alle $p$ Komponenten, die mit $j$ bzw. $k$ ein gemeinsames Ion haben $(i=1$ bis $p)$.

Die betrachteten Ketten sind vom Typ

$$
\mathrm{A}|\mathrm{AX}+\mathrm{BY}+\ldots| \mathrm{X} \text {. }
$$

Es existieren verschiedene Vorschläge zur Bezeichnung dieses Typs der reversiblen galvanischen Kette. Der Name Bildungskette ruft im Deutschen etwas unglückliche Assoziationen hervor. Wir bevorzugen daher im folgenden die Bezeichnung Chemische Kette, die auf die zugrunde liegende chemische Reaktion hinweist. Die Chemische Kette findet man auch im französischen Sprachgebrauch wieder, was man bei BRUNEAUX et al. ${ }^{5}$ nachlesen kann.

Wir wollen nun Gl. (3) in expliziter Form für ein beliebig zusammengesetztes System angeben. Dazu untersuchen wir die Funktion $\psi_{j}{ }^{\text {id }}$, die sich für jede Komponente des Systems berechnen läßt.

\section{Bestimmung der Funktion $\psi_{j}^{\text {id }}$}

Wir betrachten das System

$$
\mathrm{A}_{v_{1+}} \mathrm{X}_{v_{1-}}+\mathrm{B}_{v_{2+}} \mathrm{X}_{v_{2-}},
$$

also eine Elektrolytschmelze bei gegebener Temperatur und gegebenen Druck, die zwei Komponenten enthält. Die beiden Komponenten haben das Anion

5 M. Bruneaux, J. Hladik, Y. Pointud u. G. Morand, Electrochim. Acta 13, 1591 [1968]. gemeinsam. Nach gründlicher Durchsicht des vorhandenen experimentellen Materials hat sich herausgestellt, daß bei den EMK-Messungen an chemischen Ketten dieser Systemtyp am weitaus häufigsten untersucht wurde. Da wir unsere Betrachtungen auf Drei- und Mehrkomponentensysteme ausdehnen wollen, können wir uns hier leider nicht der von HAASE ${ }^{1}$ gewählten Bezeichnungsweise für die Zerfallszahlen anschließen. Diese Bezeichnungsweise ist für die binären Elektrolytschmelzen sehr geeignet, besonders in Hinblick auf die Grenzgesetze. Für Mehrkomponentensysteme aber, in denen mehr als zwei Komponenten ein gemeinsames Ion enthalten können, wird sie undurchschaubar. Wir wählen daher die Bezeichnung der Zerfallszahlen der Komponenten in der in Gl. (5) angegebenen Form. Ein Molekül der Komponente 1 zerfällt also in $v_{1+}$ Kationen und $v_{1}$ - Anionen, ein Molekül der Komponente 2 in $v_{2}$. Kationen und $v_{2}$. Anionen usw. Daraus folgen die Beziehungen

$$
\begin{aligned}
& v_{1}=v_{1+}+v_{1-}, \\
& v_{2}=v_{2+}+v_{2-}
\end{aligned}
$$

oder allgemein

$$
v_{j}=\sum_{\alpha} v_{j \alpha} \quad(\alpha=+,-) .
$$

$v_{j}$ ist die Summe der Zerfallszahlen für die Komponente $j$.

Eine ideale Elektrolytschmelze ist definiert durch die Gleichung

$$
\mu_{j \alpha}=\mu_{0 j \alpha}+R T \ln \left(\bar{x}_{j \alpha}+\sum_{l=1}^{p} \bar{x}_{l \alpha}\right),
$$

wobei $\mu_{j a}$ bzw. $\mu_{0 j a}$ das chemische Potential der Ionensorte $\alpha$ der Komponente $j$ in der Mischung bzw. der hypothetisch reinen flüssigen Ionenart ist. $\bar{x}_{j \alpha}$ ist der wahre Molenbruch der Ionensorte $\alpha$ der Komponente $j ; \bar{x}_{l a}$ der der Komponente $l$, wobei $l$ die Komponenten bezeichnet, die mit $j$ ein gemeinsames Ion haben. Gehen wir zur Grenze $x_{j} \rightarrow 1$ über, so erhalten wir aus Gl. ( 7 a)

$$
\mu_{j \alpha}^{0}=\mu_{0 j \alpha}+R T \ln \left(\bar{x}_{j \alpha}^{0}+\sum_{l=1}^{p} \bar{x}_{l \alpha}^{0}\right) .
$$

Dabei bedeutet

und

$$
\begin{aligned}
& \bar{x}_{j \alpha}^{0}=\lim _{x_{j} \rightarrow 1} \bar{x}_{j \alpha} \\
& \bar{x}_{l \alpha}^{0}=\lim _{x_{j} \rightarrow 1} \bar{x}_{l \alpha} .
\end{aligned}
$$

Aus Definition ( $7 \mathrm{~d})$ geht hervor, da $\beta \bar{x}_{l \alpha}^{0}$ immer verschwindet. Damit wird aus Gl. ( $7 \mathrm{~b}$ )

$$
\mu_{j \alpha}^{0}=\mu_{0 j \alpha}+R T \ln \bar{x}_{j \alpha}^{0} .
$$


Mit den Beziehungen

und

$$
\mu_{j}=\sum_{\alpha} v_{j \alpha} \mu_{j \alpha}
$$

und den Gln. (7 a) und (7 e) wird aus Gl. (2)

$$
\psi_{j}=\sum_{\alpha} \boldsymbol{v}_{j \alpha} \ln \frac{\bar{x}_{j \alpha}+\sum_{l=1}^{p} \bar{x}_{l \alpha}}{\bar{x}_{j \propto}^{0}} .
$$

Für unser in Gl. (5) angegebenes System erhalten wir aus Gl. (8)

$$
\psi_{1}=v_{1+} \ln \frac{\bar{x}_{1+}}{\bar{x}_{1+}^{0}}+v_{1-} \ln \frac{\bar{x}_{1-}+\bar{x}_{2-}}{\bar{x}_{1-}^{0}}
$$

und $\quad \psi_{2}=v_{2+} \ln \frac{\bar{x}_{2+}}{\bar{x}_{2+}^{0}}+v_{2-} \ln \frac{\bar{x}_{1-}+\bar{x}_{2-}}{\bar{x}_{2-}^{0}}$.

Die wahren Molenbrüche $\bar{x}_{j \alpha}$ hängen mit den stöchiometrischen Molenbrüchen $x_{j}$ wie folgt zusammen

$$
\bar{x}_{j \alpha}=\frac{v_{j \alpha} x_{j} \alpha_{j}}{1+\sum_{i=1}^{n} x_{i} \alpha_{i}\left(v_{i}-1\right)} .
$$

$\alpha_{j}$ ist dabei der Dissoziationsgrad der Komponente $j$. Mit Gl. (11) finden wir für unser System (5)

$$
\bar{x}_{1+}=\frac{v_{1+} x_{1} \alpha_{1}}{1+x_{1} \alpha_{1}\left(v_{1}-1\right)+x_{2} \alpha_{2}\left(v_{2}-1\right)}
$$

sowie

$$
\bar{x}_{1-}+\bar{x}_{2-}=\frac{v_{1-} x_{1} \alpha_{1}+v_{2}-x_{2} \alpha_{2}}{1+x_{1} \alpha_{1}\left(v_{1}-1\right)+x_{2} \alpha_{2}\left(v_{2}-1\right)} .
$$

Für die Grenzwerte der Gln. (12) und (13) ergibt sich aus Gl. (8)

$$
\bar{x}_{1+}^{0}=\lim _{x_{1} \rightarrow 1} \bar{x}_{1+}=\frac{v_{1+} \alpha_{1}{ }^{0}}{1+\left(v_{1}-1\right) \alpha_{1}{ }^{0}}
$$

und

$$
\bar{x}_{1-}^{0}=\lim _{x_{1} \rightarrow 1} \bar{x}_{1-}=\frac{v_{1-} a_{1}{ }^{0}}{1+\left(v_{1}-1\right) \alpha_{1}{ }^{0}} .
$$

$\alpha_{1}{ }^{0}$ bzw. allgemein $\alpha_{j}{ }^{0}$ bedeutet den Dissoziationsgrad der reinen flüssigen Komponente $j$.

Mit den Gln. (12), (13), (14) und (15) kann man den Ausdruck (9) für $\psi_{1}$ bestimmen. In ihm ist nun noch der Dissoziationsgrad $\alpha$ enthalten. Wollen wir $\psi_{1}$ explizit berechnen, so müssen wir vollständige Dissoziation voraussetzen. Dies ist keine Einschränkung für unsere Berechnungen, da nur $\psi_{1}{ }^{\text {id }}$ in den Aktivitätskoeffizienten eingeht. Vollständige Dissoziation bedeutet einerseits

$$
\alpha_{j}=\alpha_{j}^{0}=1,
$$

andererseits

$$
\psi_{j}=\psi_{j}^{\mathrm{id}} .
$$

Führt man Gl. (16) in die Gln. (12) bis (15) ein und faßt man entsprechend Gl. (9) zusammen, so ergibt sich

$$
\begin{gathered}
\frac{\bar{x}_{1+}}{\bar{x}_{1+}^{0}}=\frac{v_{1} x_{1}}{v_{1} x_{1}+v_{2} x_{2}} \\
\text { und } \frac{\bar{x}_{1-}+\bar{x}_{2-}}{x_{1-}^{0}}=\frac{\left[x_{1}+\left(v_{2-} / v_{1-}\right) x_{2}\right] v_{1}}{v_{1} x_{1}+v_{2} x_{2}} .
\end{gathered}
$$

Für die Funktion $\psi_{1}{ }_{1}^{\text {id }}$ erhält man aus den Gln. (9), (18) und (19) unter Berücksichtigung von Gl. (6)

$$
\psi_{1}^{\text {id }}=\ln \frac{v_{1}^{v_{1}} x_{1} v_{1+}\left[x_{1}+\left(v_{2}-/ v_{1-}\right) x_{2}\right]{ }^{v_{1-}}}{\left(v_{1} x_{1}+v_{2} x_{2}\right)^{v_{1}}} .
$$

Auf ganz analogem Weg berechnet sich $\psi_{2}{ }^{\text {id }}$ für die Komponente 2 des Systems (5)

$$
\psi_{2}{ }^{\mathrm{id}}=\ln \frac{v_{2} \nu_{2} x_{2} v_{2+}\left[x_{2}+\left(v_{1}-/ v_{2}\right) x_{1}\right] v_{2-}}{\left(v_{1} x_{1}+v_{2} x_{2}\right)^{v_{2}}} .
$$

Die so gewonnenen $\psi_{1}{ }^{\text {id }}$ und $\psi_{2}{ }^{\text {id }}$ charakterisieren eine Elektrolytschmelze, die wie das System (5) aufgebaut - dies ist ein großer Teil der in der Literatur untersuchten Ketten - und vollständig dissoziiert ist.

Es werden nun andere Systemtypen untersucht, so daß wir in der Lage sein werden, die allgemeine Formel für $\psi_{j}^{\text {id }}$ abzuleiten. Wir betrachten zuerst das einfachste System, das aus zwei Komponenten besteht und keine gemeinsamen Ionen enthält. Es ist in unserer Schreibweise wie folgt aufgebaut

$$
\mathrm{A}_{v_{1+}} \mathrm{X}_{v_{1-}}+\mathrm{B}_{v_{2+}} \mathrm{Y}_{v_{2-}} \text {. }
$$

Für dieses System finden wir mit Gl. (8)

$$
\psi_{1}=v_{1+} \ln \left(\bar{x}_{1+} / \bar{x}_{1+}^{0}\right)+v_{1-} \ln \left(\bar{x}_{1-} / \bar{x}_{1-}^{0}\right)
$$

und

$$
\psi_{2}=v_{2+} \ln \left(\bar{x}_{2+} / \bar{x}_{2+}^{0}\right)+v_{2-} \ln \left(\bar{x}_{2-} / \bar{x}_{2-}^{0}\right) .
$$

Mit Hilfe der Gln. (8), (11), (16) und (17) erhält man aus Gl. (23 a)

$$
\psi_{1}^{\mathrm{id}}=\ln \frac{v_{1} v_{1} x_{1}{ }^{v_{1}}}{\left(v_{1} x_{1}+v_{2} x_{2}\right) v_{2}}
$$

und aus Gl. (23 b)

$$
\psi_{2}^{\mathrm{id}}=\ln \frac{v_{2}^{\nu_{2}} x_{2} v_{2}}{\left(v_{1} x_{1}+v_{2} x_{2}\right) v_{2}} .
$$

Die Gln. (24) und (25) charakterisieren das System $(22)^{6}$.

${ }^{6}$ Diese Gleichungen stimmen mit den von HAAsE ${ }^{1}$ für binäre Elektrolytschmelzen angegebenen Ausdrücken überein, wenn man spezielle Systeme einsetzt. 
Gehen wir nun einen Schritt weiter und betrachten eine Elektrolytschmelze mit drei Komponenten. Das System habe die Form

$$
\mathrm{A}_{v_{1+}} \mathrm{X}_{v_{1-}}+\mathrm{B}_{v_{2+}} \mathrm{Y}_{v_{2-}}+\mathrm{C}_{v_{3+}} \mathrm{Z}_{v_{3-}} .
$$

Die Komponenten haben also keine gemeinsamen Ionen. Aus Gl. (8) folgt

$$
\begin{aligned}
\psi_{1} & =v_{1+} \ln \left(\bar{x}_{1+} / \bar{x}_{1+}^{0}\right)+v_{1-} \ln \left(\bar{x}_{1-} / \bar{x}_{1-}^{0}\right) \\
\psi_{2} & =v_{2+} \ln \left(\bar{x}_{2+} / \bar{x}_{2+}^{0}\right)+v_{2-} \ln \left(\bar{x}_{2-} / \bar{x}_{2-}^{0}\right), \\
\text { und } \psi_{3} & =v_{3+} \ln \left(\bar{x}_{3+} / \bar{x}_{3+}^{0}\right)+v_{3-} \ln \left(\bar{x}_{3-} / \bar{x}_{3-}^{0}\right) .
\end{aligned}
$$

Mit den oben angegebenen Gleichungen finden wir die gesuchten Funktionen

$$
\begin{aligned}
& \psi_{1}{ }^{\mathrm{id}}=\ln \frac{v_{1}{ }^{\nu_{1}} x_{1}{ }^{\nu_{1}}}{\left(v_{1} x_{1}+v_{2} x_{2}+v_{3} x_{3}\right)^{v_{1}}}, \\
& \psi_{2}{ }^{\mathrm{id}}=\ln \frac{v_{2} v_{2} x_{2}{ }^{\nu_{2}}}{\left(v_{1} x_{1}+v_{2} x_{2}+v_{3} x_{3}\right)^{\nu_{2}}}
\end{aligned}
$$

und

$$
\psi_{3}{ }^{\mathrm{id}}=\ln \frac{v_{3}{ }^{{ }_{3}} x_{3}{ }^{\nu_{3}}}{\left(v_{1} x_{1}+\nu_{2} x_{2}+\nu_{3} x_{3}\right)^{\nu_{3}}} .
$$

Die Gln. (30), (31) und (32) beschreiben die vollständig dissoziierte ideale Elektrolytschmelze vom Typ (26).

Nun untersuchen wir eine Elektrolytschmelze mit drei Komponenten, die gemeinsame Kationen und Anionen enthalten. Der Systemtyp sei beschrieben durch

$$
\mathrm{A}_{v_{1+}} \mathrm{X}_{v_{1-}}+\mathrm{B}_{v_{2+}} \mathrm{X}_{v_{2-}}+\mathrm{A}_{v_{3+}} \mathrm{Y}_{v_{3-}} .
$$

Die Komponente 1 hat also das Kation mit der Komponente 3 gemeinsam, das Anion mit der Komponente 2 gemeinsam. Mit Gl. (8) ist

$$
\begin{aligned}
\psi_{1}= & v_{1+} \ln \left[\left(\bar{x}_{1+}+\bar{x}_{3+}\right) / \bar{x}_{1+}^{0}\right] \\
& +v_{1-} \ln \left[\left(\bar{x}_{1-}+\bar{x}_{2-}\right) / \bar{x}_{1-}^{0}\right], \\
\psi_{2}= & v_{2+} \ln \left(\bar{x}_{2+} / \bar{x}_{2+}^{0}\right) \\
& +v_{2-} \ln \left[\left(\bar{x}_{1+}+\bar{x}_{2-}\right) / \bar{x}_{1-}^{0}\right]
\end{aligned}
$$

und $\psi_{3}=v_{3+} \ln \left[\left(\bar{x}_{1+}+\bar{x}_{3+}\right) / \bar{x}_{3+}^{0}\right]$

$$
+v_{3-} \ln \left(\bar{x}_{3-} / \bar{x}_{3-}^{0}\right) \text {. }
$$

Da in diesen Gleichungen wieder die Dissoziationsgrade der drei Komponenten enthalten sind, gehen wir wie oben zur vollständigen Dissoziation über und erhalten für die entsprechenden Funktionen der idealen Elektrolytschmelze vom Typ (33)

$$
\begin{aligned}
& \psi_{1}^{\mathrm{id}}=\ln \frac{v_{1} v_{1}\left[x_{1}+\left(v_{3+} / v_{1+}\right) x_{3}\right] v_{1+}\left[x_{1}+\left(v_{2-} / v_{1-}\right) x_{2}\right] v_{1-}}{\left(v_{1} x_{1}+v_{2} x_{2}+v_{3} x_{3}\right) v_{1}}, \\
& \psi_{2}^{\text {id }}=\ln \frac{v_{2} v_{2}\left[x_{2}+\left(v_{1-} / v_{2-}\right) x_{1}\right] v_{2-} x_{2} v_{2+}}{\left(v_{1} x_{1}+v_{2} x_{2}+v_{3} x_{3}\right) v_{2}}
\end{aligned}
$$

und

$\psi_{3}{ }^{\text {id }}=\ln \frac{v_{3}{ }^{v_{3}}\left[x_{3}+\left(v_{1}+v_{3}\right) x_{1}\right]^{v_{3+}} x_{3}{ }^{\nu_{3-}}}{\left(v_{1} x_{1}+v_{2} x_{2}+v_{3} x_{3}\right)^{v_{3}}}$.

Der Vergleich der bisher gewonnenen Ergebnisse (20), (21), (24), (25), (30) bis (32) und (37) bis (39) erlaubt nun, eine gemeinsame Formel abzuleiten, die alle Ergebnisse in einer Gleichung beschreibt. Wir bezeichnen wie bisher mit $j$ eine beliebige Komponente des Systems, mit $l$ die Komponente, die mit $j$ ein gemeinsames Ion hat und mit dem Index $\alpha$ das Kation $(+)$ bzw. Anion $(-)$. Die Zusammenfassung ergibt

$$
\psi_{j}^{\mathrm{id}}=\ln \frac{v_{j}{ }^{\nu_{j}} \prod_{j \alpha}\left[x_{j}+\left(v_{l \alpha} / v_{j \alpha}\right) x_{l}\right]^{v_{j a}}}{\left(\sum_{i=1}^{n} v_{i} x_{i}\right)^{v_{j}}} .
$$

$v_{l a}$, die Zerfallszahl des Ions, das die Komponente $l$ mit der Komponente $j$ gemeinsam hat, ist definitionsgemäß Null für solche Ionen, die in der Komponente $j$ nicht enthalten sind. Das Produkt im Zähler der Formel erstreckt sich über die Ionen der Komponente $j$. Leitet man nun umgekehrt aus Gl. (40) die Ergebnisse der bisher betrachteten Systeme $\mathrm{ab}$, so sieht man, daß die Formel richtig ist für solche Systeme, in denen nicht mehr als zwei Komponenten ein gemeinsames Ion haben. Wenn mehr als zwei Komponenten ein gemeinsames Ion haben, so muß Gl. (40) erweitert werden. Zu diesem Zweck betrachten wir eine Elektrolytschmelze mit vier Kom. ponenten.

$\mathrm{A}_{v_{1+}} \mathrm{X}_{v_{1-}}+\mathrm{A}_{v_{2+}} \mathrm{Y}_{v_{2-}}+\mathrm{A}_{v_{3+}} \mathrm{Z}_{v_{3-}}+\mathrm{B}_{v_{4+}} \mathrm{Y}_{v_{4-}}$.

Dies ist ein Beispiel, in dem mehr als zwei Komponenten ein gemeinsames Ion haben. Zwar ist ein Vierkomponentensystem vom Experiment her ungewöhnlich, aber nicht ausgeschlossen. Es diene hier symbolisch zur Veranschaulichung der nötigen Erweiterung unserer Gl. (40). Die Komponenten 1, 2 und 3 haben ein gemeinsames Kation, die Komponenten 2 und 4 ein gemeinsames Anion. Aus Gl. (8) folgt für dieses System

$$
\begin{aligned}
\psi_{1}= & v_{1+} \ln \left[\left(\bar{x}_{1+}+\bar{x}_{2+}+\bar{x}_{3+}\right) / \bar{x}_{1+}^{0}\right] \\
& +v_{1-} \ln \left(\bar{x}_{1-} / \bar{x}_{1}^{0}\right), \\
\psi_{2}= & v_{2+} \ln \left[\left(\bar{x}_{1+}+\bar{x}_{2+}+\bar{x}_{3+}\right) / \bar{x}_{2+}^{0}\right] \\
& +v_{2-} \ln \left[\left(\bar{x}_{2-}+\bar{x}_{4-}\right) / \bar{x}_{2-}^{0}\right], \\
\psi_{3}= & v_{3+} \ln \left[\left(\bar{x}_{1+}+\bar{x}_{2+}+\bar{x}_{3+}\right) / \bar{x}_{3+}^{0}\right] \\
& +v_{3-} \ln \left(\bar{x}_{3-} / \bar{x}_{3-}^{0}\right) \\
\psi_{4}= & v_{4+} \ln \left(\bar{x}_{4+} / \bar{x}_{4+}^{0}\right] \\
& +v_{4-} \ln \left[\left(\bar{x}_{2-}+\bar{x}_{4-}\right) / \bar{x}_{4}^{0}\right] .
\end{aligned}
$$$$
\text { und } \quad \psi_{4}=v_{4+} \ln \left(\bar{x}_{4+} / \bar{x}_{4+}^{0}\right]
$$ 
Mit den Gln. (8), (11), (16) und (17) erhält man aus Gl. (42)

aus Gl. (43)

$$
\psi_{1}{ }^{\mathrm{id}}=\ln \frac{v_{1}^{v_{1}}\left[x_{1}+\left(v_{2}+v_{1+}\right) x_{2}+\left(v_{3}+v_{1+}\right) x_{3}\right]^{v_{1+}} x_{1}{ }^{v_{1-}}}{\left(v_{1} x_{1}+v_{2} x_{2}+v_{3} x_{3}+v_{4} x_{4}\right) v_{1}},
$$

$$
\psi_{2} \mathrm{id}=\ln \frac{v_{2} \nu_{2}\left[x_{2}+\left(v_{1+} / v_{2+}\right) x_{1}+\left(v_{3} / v_{2+}\right) x_{3}\right]^{v_{2+}}\left[x_{2}+\left(v_{4-} / v_{2-}\right) x_{4}\right]^{v_{2-}}}{\left(v_{1} x_{1}+v_{2} x_{2}+v_{3} x_{3}+v_{4} x_{4}\right)^{\nu_{2}}},
$$

aus Gl. (44)

und aus Gl. (45)

$$
\psi_{3}{ }^{\mathrm{id}}=\ln \frac{v_{3^{\nu_{3}}}\left[x_{3}+\left(v_{1+} / v_{3+}\right) x_{1}+\left(v_{2+} / v_{3+}\right) x_{2}\right]^{v_{3+}} x_{3} \nu_{3-}}{\left(v_{1} x_{1}+v_{2} x_{2}+v_{3} x_{3}+v_{4} x_{4}\right)^{\nu_{3}}}
$$

$$
\psi_{4}^{\mathrm{id}}=\ln \frac{v_{4}^{v_{4}} x_{4}^{v_{4+}}\left[x_{4}+\left(v_{2}-/ v_{4}\right) x_{2}\right]^{v_{4-}}}{\left(v_{1} x_{1}+v_{2} x_{2}+v_{3} x_{3}+v_{4} x_{4}\right)^{v_{4}}} .
$$

Diese vier Funktionen beschreiben die vollständig dissoziierte ideale Elektrolytschmelze vom Typ (41). Die komplizierteste davon ist Gl. (47). Man sieht, daß sich diese Ausdrücke unter bestimmten Bedingungen sehr vereinfachen. Handelt es sich z. B. um Elektrolyte, für die gilt $v_{1}=v_{2}=v_{3}=v_{4}$, so kürzt sich der Faktor $v_{j}^{v_{j}}$ heraus und der Nenner wird Eins. Überhaupt treten bei den meisten einfachen Elektrolyten, die normalerweise in der Literatur untersucht werden, erhebliche Vereinfachungen auf, wie weiter unten gezeigt wird. Trotzdem muß die Ableitung in voller Allgemeinheit durchgeführt werden, damit man nun den endgültigen Charakter der allgemeinen Formulierung von $\psi_{j}{ }^{\text {id }}$ erkennen kann. Gl. (47) zeigt, daß man den Zähler von Gl. (40) um die möglichen Summanden erweitern muß. Für $\psi_{j}{ }^{\text {id }}$ ergibt sich allgemein

$$
\psi_{j}^{\mathrm{id}}=\ln \frac{v_{j}^{v_{j}} \prod_{j \alpha}\left(x_{j}+\sum_{l=1}^{p}\left(v_{l a} / v_{j \alpha}\right) x_{l}\right)^{v_{j \alpha}}}{\left(\sum_{i=1}^{n} v_{i} x_{i}\right)^{v_{j}}} .
$$

Wie in Gl. (40) erstreckt sich das Produkt über die Ionen der Komponente $j$ und verschwindet $v_{l \alpha}$ definitionsgemäß für solche Ionen, die die Komponente $j$ nicht enthält. Die Summe erstreckt sich über alle Komponenten $l$, die mit $j$ ein gemeinsames Ion haben. Zieht man $x_{j}$ im Zähler noch in die Summe über $l$ mit hinein - ändert in diesem Sinn natürlich auch den Summationsindex - , so erscheint die Gleichung formal zwar noch einfacher, macht die Benutzung der Formel aber unnötig schwieriger, besonders bei solchen Systemen, die noch mit Gl. (40) erfaßt werden können. Da es sich bei diesen Systemen um die große Mehrzahl der untersuchten Elektrolytschmelzen handelt, wollen wir diese formale Änderung nicht einführen. Mit Gl. (50) wird nun auch ein Dreikomponentensystem erfaßt, in dem alle drei
Komponenten z. B. ein gemeinsames Kation haben, was mit Gl. (40) nicht mehr möglich war. Das System habe die Form

$$
\mathrm{A}_{v_{1+}} \mathbf{X}_{v_{1-}}+\mathrm{A}_{v_{2+}} \mathrm{Y}_{v_{2-}}+\mathrm{A}_{v_{3+}} \mathrm{Z}_{v_{3-}} .
$$

Für $\psi_{1}{ }^{\text {id }}$ ergibt sich aus Gl. (50) direkt

$\psi_{1}{ }^{\mathrm{id}}=\ln \frac{v_{1} v_{1}\left[x_{1}+\left(v_{2}+/ v_{1+}\right) x_{2}+\left(v_{3}+/ v_{1+}\right) x_{3}\right]^{v_{1+}} x_{1}{ }^{\nu_{1-}}}{\left(v_{1} x_{1}+v_{2} x_{2}+v_{3} x_{3}\right)^{\nu_{1}}}$.

Dieses Beispiel demonstriert den Gebrauch von Gl. (50); $\psi_{2}{ }^{\mathrm{id}}$ und $\psi_{3}{ }^{\mathrm{id}}$ ergeben sich analog.

\section{Berechnung der Aktivitätskoeffizienten}

Wir behalten Gl. (1) als allgemeine Definition des Aktivitätskoeffizienten $f_{j}$ bei. Damit folgt aus den Gln. (1), (3) und (50)

$\ln f_{k}=\frac{F\left(\Phi-\Phi_{0}\right)}{q R T}-\ln \frac{v_{k}{ }^{v_{k}} \prod_{k \alpha}\left(x_{k}+\sum_{l=1}^{p}\left(v_{l \alpha} / v_{k \alpha}\right) x_{l}\right)^{v_{k \alpha}}}{\left(\sum_{i=1}^{n} v_{i} x_{i}\right)^{v_{k}}}$.

Mit dieser Gleichung läßt sich aus EMK-Messungen an chemischen Ketten der Aktivitätskoeffizient der Komponente $k$ - im obigen Sinne - jeder Elektrolytschmelze, die sich aus beliebig vielen Elektrolyten zusammensetzt, bestimmen. Hat man den Aktivitätskoeffizienten einer Komponente experimentell ermittelt, so lassen sich die anderen Aktivitätskoeffizienten auf folgendem Weg berechnen.

Die Gibbs-Duhemsche Beziehung ganz allgemein lautet

$$
\sum_{i=1}^{n} x_{i} D \mu_{i}=0 .
$$

$D \mu_{i}$ ist eine infinitesimale Änderung des chemischen Potentials der Komponente $i$ durch die Änderung 
der Zusammensetzung der Elektrolytschmelze bei konstanter Temperatur und konstantem Druck ${ }^{3}$.

Aus der Definition (1) folgt mit Gl. (2)

$$
\mu_{j}=\mu_{0 j}+R T \psi_{j}^{\mathrm{id}}+R T \ln f_{j} .
$$

Setzen wir Gl. (55) in Gl. (54) ein, so erhalten wir

$$
R T \sum_{i=1}^{n} x_{i} D \psi_{i}^{\mathrm{id}}+R T \sum_{i=1}^{n} x_{i} D \ln f_{i}=0 .
$$

Wegen der Identität

$$
\sum_{i=1}^{n} x_{i}=1
$$

gibt es nur $(n-1)$ unabhängige stöchiometrische Molenbrüche. Wählen wir $x_{1}$ als abhängigen Molenbruch, so hat die infinitesimale Größe im ersten Term der Gl. (56) folgende Gestalt

$$
\begin{aligned}
& D \psi_{i}^{\mathrm{id}}=\left(\frac{\partial \psi_{i}^{\mathrm{id}}}{\partial x_{2}}\right)_{T, P, x_{3}, x_{4} \ldots} \mathrm{d} x_{2}+ \\
&+\left(\frac{\partial \psi_{i}^{\mathrm{id}}}{\partial x_{3}}\right)_{T, P, x_{2}, x_{4} \ldots} \mathrm{d} x_{3}+\ldots
\end{aligned}
$$

Multipliziert man Gl. (58) mit $x_{i}$ und summiert über alle $i$, so verschwindet der Ausdruck für alle Systeme, was sich durch Einsetzen der entsprechenden Gleichungen elementar verifizieren läßt. Damit folgt aus Gl. (56)

$$
\sum_{i=1}^{n} x_{i} D \ln f_{i}=0
$$

Mit Hilfe dieser Form der Gibbs-Duhemschen Beziehung und der Identität (58) können die Aktivitätskoeffizienten aller Komponenten eines Systems prinzipiell ermittelt werden, wenn man einen Aktivitätskoeffizienten experimentell bestimmt hat. Für binäre Systeme ist das gezeigt worden ${ }^{1}$. Für eine Schmelze mit mehr als zwei Komponenten muß man zusätzlich das bekannte Gleichungssystem anwenden, das durch die Integrabilitätsbedingungen der $\mathrm{Zu}$ standsfunktionen, in diesem Fall die freie Enthalpie $G$, geliefert wird.

\section{Diskussion der Ergebnisse}

An Hand von Literaturwerten soll nun die oben gewonnene Gl. (53) diskutiert werden. Als erstes betrachten wir ein System, das aus zwei Komponenten besteht und keine gemeinsamen Ionen hat, wie

7 Ch. Dijkhuis, R. Dijkhuis u. G. J. Janz, Chem. Rev. 68, 253 [1968]. in Gl. (22) schematisch dargestellt. Man spricht in diesem Fall von einem reziproken Salzpaar wie z. B. $\mathrm{KBr}+\mathrm{AgCl}$. In Übereinstimmung mit den binären Elektrolytschmelzen wollen wir bei der Auswertung die Komponente $k$ immer als die zweite Komponente auffassen. $f_{2}$ ist also der Aktivitätskoeffizient der Komponente, für deren Ionen die Elektroden reversibel sind, d. h. $f_{2}$ ist der Aktivitätskoeffizient derjenigen Komponente, die im reinen Zustand in einer Kette mit der EMK $\Phi_{0}$ vorkommt.

Mit $q=-1$ und $v_{1}=v_{2}=2$ erhält man aus Gl. (53) für das System $\operatorname{KBr}(1)+\operatorname{AgCl}(2)$

$$
\ln f_{2}=\frac{F\left(\Phi_{0}-\Phi\right)}{R T}-2 \ln x_{2} .
$$

Leider ist uns in der Literatur kein solches Beispiel bekannt. Die neueste umfangreiche Bibliographie für EMK-Ketten von BRUNEAUX et al. ${ }^{5}$ enthält ebensowenig ein solches einfaches System wie die zusammenfassende Arbeit von DijkHUIs und JANZ ${ }^{7}$.

In der Theorie der Salzschmelzen definiert man Aktivitätskoeffizienten $\gamma_{j}$ häufig durch

$$
\mu_{j}=\mu_{0 j}+R T \ln x_{j}+R T \ln \gamma_{j} .
$$

Diese Auswertung wird zeigen, daß diese Definition nur für einige spezielle Systeme sinnvoll ist. Der Vergleich mit Gl. (55) zeigt, daß der Verlauf der Aktivitätskoeffizienten in Abhängigkeit von der Konzentration von Gl. (61) abweicht. Dies wird am folgenden Beispiel gezeigt. BLoom und $\mathrm{WELCH}^{8}$ untersuchten das etwas kompliziertere reziproke Salzpaar $\mathrm{NaCl}+\mathrm{PBr}_{2}$ bei $900 \mathrm{~K}$ und $1000 \mathrm{~K}$. Aus Gl. (53) folgt mit $q=-\frac{1}{2}, v_{1}=2$ und $v_{2}=3$

$$
\ln f_{2}=\frac{2 F\left(\Phi_{0}-\Phi\right)}{R T}-\ln \frac{27 x_{2}{ }^{3}}{\left(2+x_{2}\right)^{3}} .
$$

Die Ergebnisse dieser Gleichung sind in Tab. 1 wiedergegeben. In Spalte 1 ist der Molenbruch der Komponente $2\left(\mathrm{PbBr}_{2}\right)$ tabelliert, in Spalte 2 die nach Gl. (62) berechneten Aktivitätskoeffizienten bei $900 \mathrm{~K}$, in Spalte 3 die von Bloom und Welch berechneten bei der gleichen Temperatur, in den Spalten 4 und 5 die analogen Größen für $1000 \mathrm{~K}$ und in Spalte 6 befinden sich die Werte der Abweichfunktion

$$
r_{2}=27 x_{2}^{2} /\left(2+x_{2}\right)^{3} \text {. }
$$

Diese Funktion beschreibt das Verhältnis der von uns berechneten Aktivitätskoeffizienten zu denen,

\footnotetext{
${ }^{8}$ H. Bloom u. B. J. Welch, Trans. Faraday Soc. 59, 410
} [1963]. 


\begin{tabular}{|c|c|c|c|c|c|c|c|c|c|c|c|}
\hline \multirow{3}{*}{$x_{2}$} & \multirow{2}{*}{\multicolumn{4}{|c|}{$\begin{array}{c}\mathrm{NaCl}+\mathrm{PbBr}_{2} \\
1000 \mathrm{~K}\end{array}$}} & \multirow{3}{*}{$r_{1}$} & \multicolumn{6}{|c|}{$\mathrm{KCl}+\mathrm{PbCl}_{2}$} \\
\hline & & & & & & $\gamma_{2}$ & 773 & & 97 & & $r_{2}$ \\
\hline & $f_{2}$ & $\gamma_{2}$ & $f_{2}$ & $\gamma_{2}$ & & $f_{2}$ & $f_{2}$ & $\gamma_{2}$ & $f_{2}$ & $\gamma_{2}$ & \\
\hline 1,0 & 1,00 & 1,00 & 1,00 & 1,00 & 1,000 & 1,000 & 1,000 & 1,000 & 1,000 & 1,000 & 1,000 \\
\hline 0,9 & 1,01 & - & 1,01 & - & 0,897 & - & 1,082 & 1,081 & 1,008 & 1,007 & 0,999 \\
\hline 0,8 & 1,04 & 1,03 & 1,04 & 1,03 & 0,787 & 0,990 & 0,913 & 0,909 & 0,951 & 0,947 & 0,996 \\
\hline 0,7 & 1,11 & 1,07 & 1,09 & 1,07 & 0,672 & 0,973 & 0,652 & 0,646 & 0,818 & 0,811 & 0,991 \\
\hline 0,6 & 1,24 & 1,20 & 1,19 & 1,17 & 0,553 & 0,976 & 0,528 & 0,519 & 0,688 & 0,676 & 0,983 \\
\hline 0,5 & 1,50 & 1,41 & 1,38 & 1,31 & 0,432 & 0,945 & 0,399 & 0,388 & 0,535 & 0,520 & 0,972 \\
\hline 0,4 & 2,02 & 1,76 & 1,73 & 1,61 & 0,313 & 0,901 & 0,265 & 0,254 & 0,392 & 0,375 & 0,957 \\
\hline 0,3 & 2,84 & 2,39 & 2,38 & 2,07 & 0,200 & 0,856 & 0,164 & 0,154 & 0,301 & 0,282 & 0,938 \\
\hline 0,2 & 3,88 & 3,43 & 3,34 & 2,84 & 0,101 & 0,867 & 0,129 & 0,109 & 0,245 & 0,224 & 0,913 \\
\hline 0,1 & & & & & 0,029 & & 0,090 & 0,080 & 0,204 & 0,180 & 0,882 \\
\hline 0,0 & & & & & 0,000 & & & & & & 0,844 \\
\hline
\end{tabular}

Tab. 1. Aktivitätskoeffizienten für die Systeme $\mathrm{NaCl}+\mathrm{PbBr}_{2}$ und $\mathrm{KCl}+\mathrm{PbBr}_{2}$ bei verschiedenen Temperaturen.

die man entsprechend der Definition (61) berechnen würde. Der Vergleich zeigt, daß $f_{2}$ und $\gamma_{2}$ sehr viel besser übereinstimmen. Die tatsächliche mittlere $\mathrm{Ab}$ weichung ist in Spalte 7 wiedergegeben. Auch Bloom und Welch weisen darauf hin, daß die Übertragung der Definition für die niedrigmolekularen Nichtelektrolyte auf die Elektrolytschmelzen für reziproke Salze nicht ausreicht, sondern - wie die Autoren feststellten - nur für Systeme mit gemeinsamen Ionen. Unsere Berechnungen ergeben, daß dies auch bei gemeinsamen Ionen nur in den einfachsten Fällen zutrifft. Spalte 7 zeigt, daß die Bloomsche Ableitung der Aktivitätskoeffizienten aus dem Temkinschen Modell und unsere Definition für dieses System ungefähr übereinstimmt. Wir halten aber eine Definition, die von jedem Modell unabhängig ist und für jedes beliebig zusammengesetzte System gilt, für die Auswertung der Experimente für geeigneter.

Die Bloomschen Werte des reziproken Systems $\mathrm{NaBr}+\mathrm{PbCl}_{2}$ sind nach unseren Gleichungen nicht auswertbar, da die Ionen keiner Komponente für die verwendeten Elektroden reversibel sind, d. h. es existiert für die so aufgebaute Kette kein $\Phi_{0}$.

Als nächstes untersuchen wir Systeme, die ein gemeinsames Anion haben. Fast alle in der Literatur angegebenen Systeme gehören zu dieser Klasse. Ein einfaches Beispiel ist das System $\mathrm{NaCl}+\mathrm{AgCl}$, das von PANisH et al. ${ }^{9}$ sehr sorgfältig untersucht wurde. Aus Gl. (53) folgt mit $q=-1$ und $v_{1}=v_{2}=2$

$$
\ln f_{2}=\frac{F\left(\Phi_{0}-\Phi\right)}{R T}-\ln x_{2} .
$$

9 M. B. Panish, F. F. Blankenship, W. R. Grimes u. R. F. Newton, J. Phys. Chem. 62, 1325 [1958].

10 E. J. Salstrom, J. Am. Chem. Soc. 55, 1029 [1933].
Für diesen Systemtyp stimmen also die Aktivitätskoeffizienten nach beiden Definitionen überein, wie der Vergleich mit (61) zeigt. Zum gleichen Ergebnis kommt man bei Systemen, für die wie im obigen Fall $v_{1}=v_{2}(>2)$ ist. Eins der wenigen Beispiele in der Literatur dafür ist die umfangreiche Arbeit von SAlstrom ${ }^{10}$ am System $\mathrm{ZnBr}_{2}+\mathrm{PbBr}_{2}$. Abweichungen hingegen findet man bei der großen Klasse der Systeme, für die $v_{1} \neq v_{2}$ ist. Als sehr gründlich untersuchtes Beispiel nennen wir die Arbeit von Lantratov und Alabyshev ${ }^{11}$, die u. a. das System $\mathrm{KCl}+\mathrm{PbCl}_{2}$ bei verschiedenen Temperaturen untersuchten. Aus Gl. (53) folgt mit $q=-\frac{1}{2}$, $v_{1}=2, v_{2}=3, v_{1-}=1$ und $v_{2-}=2$

$$
\ln f_{2}=\frac{2 F\left(\Phi_{0}-\Phi\right)}{R T}-\ln \frac{27 x_{2}\left(1+x_{2}\right)^{2}}{4\left(2+x_{2}\right)^{3}} .
$$

In Tab. 1 sind die Resultate dieser Gleichung aufgeführt. In Spalte 8 sind die nach Gl. (65) berechneten Aktivitätskoeffizienten für $500{ }^{\circ} \mathrm{C}=773,2 \mathrm{~K}$, in Spalte 9 die von Lantratov und Alabyshev angegebenen für die gleiche Temperatur, in den Spalten 10 und 11 die analogen Größen für $700{ }^{\circ} \mathrm{C}=973,2 \mathrm{~K}$. Spalte 12 enthält die Abweichfunktion

$$
r_{2}=27\left(1+x_{2}\right)^{2} /\left[4\left(2+x_{2}\right)^{3}\right] .
$$

Der Vergleich der entsprechenden Größen zeigt, daß die Abweichung zwar prinzipiell, aber nicht sehr groß ist. Dies erklärt sich dadurch, daß die kompliziert aussehende Abweichfunktion nahe bei Eins bleibt. Die Abweichfunktionen $r_{1}$ und $r_{2}$ sind in Abb. 1 dargestellt. Man sieht, daß die Abweichung der Aktivitätskoeffizienten der reziproken Salzpaare

11 M. F. Lantratov u. A. F. Alabyshev, J. Appl. Chem. 26, 235 [1953]. 
vom Typ, wie sie von Bloom und Welch untersucht wurden, von der üblichen Definition erheblich ist; bei Systemen mit gemeinsamen Anionen fällt die Abweichung weniger ins Gewicht. Man darf dabei aber nicht vergessen, daß ein prinzipieller theoretischer Unterschied zwischen beiden Definitionen besteht.

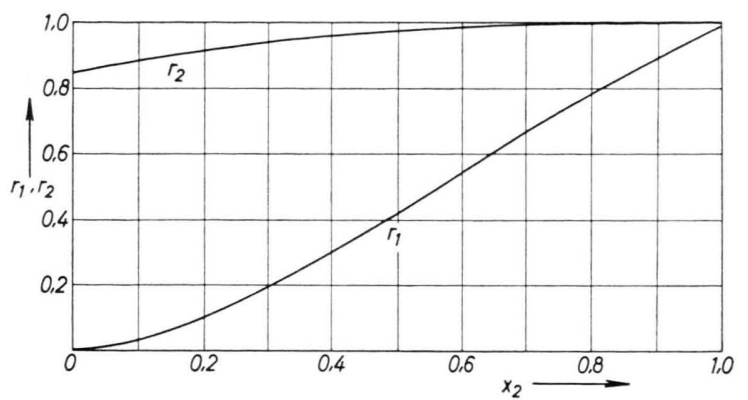

Abb. 1. Die Abweichfunktionen $r_{1}$ und $r_{2}$ in Abhängigkeit vom stöchiometrischen Molenbruch der Komponente 2.

Für Systeme mit zwei Komponenten, die ein gemeinsames Kation haben, gibt es in der Literatur nur wenige Beispiele. Die Systeme $\mathrm{AgJ}+\mathrm{AgBr}$ sowie $\mathrm{AgJ}+\mathrm{AgCl}$ wurden in Abhängigkeit von Temperatur und Konzentration von STERNBERG et al. ${ }^{12}$ untersucht. Wegen $v_{1}=v_{2}=2$ führt die Auswertung von Gl. (53) zu den Ergebnissen, die die Autoren auf Grund der Definition (61) erhielten. Das gleiche gilt für das System $\mathrm{AgBr}+\mathrm{AgCl}$, das von MurguLESCU und MARCHIDAN ${ }^{13}$ untersucht wurde.

$\mathrm{Zu}$ ganz anderen Resultaten gelangt man für das System $\mathrm{PbCl}_{2}+\mathrm{PbBr}_{2}$, das von SAlstrom und Hildebrand ${ }^{14}$ untersucht wurde. Mit $q=-\frac{1}{2}$, $v_{1}=v_{2}=3, \quad v_{1+}=v_{2+}=1$ und $v_{1-}=2$ folgt aus Gl. (53)

$$
\ln f_{2}=\frac{2 F\left(\Phi_{0}-\Phi\right)}{R T}-2 \ln x_{2} .
$$

\begin{tabular}{ccccc}
\hline & \multicolumn{2}{c}{$723,2 \mathrm{~K}$} & \multicolumn{2}{c}{$823,2 \mathrm{~K}$} \\
$x_{2}$ & $f_{2}$ & $\gamma_{2}$ & $f_{2}$ & $\gamma_{2}$ \\
\hline 1,0 & 1,000 & 1,000 & 1,000 & 1,000 \\
0,8 & 1,091 & 0,873 & 1,056 & 0,845 \\
0,6 & 1,277 & 0,766 & 1,188 & 0,713 \\
0,5 & 1,364 & 0,682 & 1,304 & 0,652 \\
0,45 & 1,307 & 0,588 & 1,280 & 0,576 \\
\hline
\end{tabular}

Tab. 2. Aktivitätskoeffizienten für das System $\mathrm{PbCl}_{2}+\mathrm{PbBr}_{2}$ bei verschiedenen Temperaturen.

12 S. Sternberg, J. Adorian u. L. Galasiu, Rev. Roumaine Chim. 11, 581 [1966].

13 I. G. Murgulescu u. D. M. Marchidan, Acad. Rep. Populaire Roumaine Bucuresti, Rev. Chim. 111, 47 [1958].

14 E. J. Salstrom u. J. H. Hildebrand, J. Am. Chem. Soc. 52, 4641 [1930].
Man findet mit dieser Gleichung eine positive Abweichung von der idealen Elektrolytschmelze statt der von den Autoren berechneten negativen Abweichung. Die Resultate sind in Tab. 2 aufgeführt. Spalte 1 enthält den Molenbruch des Bleibromids, Spalte 2 die nach Gl. (67) berechneten Aktivitätskoeffizienten bei $450{ }^{\circ} \mathrm{C}=723,2 \mathrm{~K}$, Spalte 3 die von Salstrom und Hildebrand angegebenen, Spalte 4 und 5 die entsprechenden Werte für $550{ }^{\circ} \mathrm{C}=823,2$ K. In Abb. 2 sind die nach Gl. (67) berechneten $f_{2}$ für verschiedene Temperaturen dargestellt.

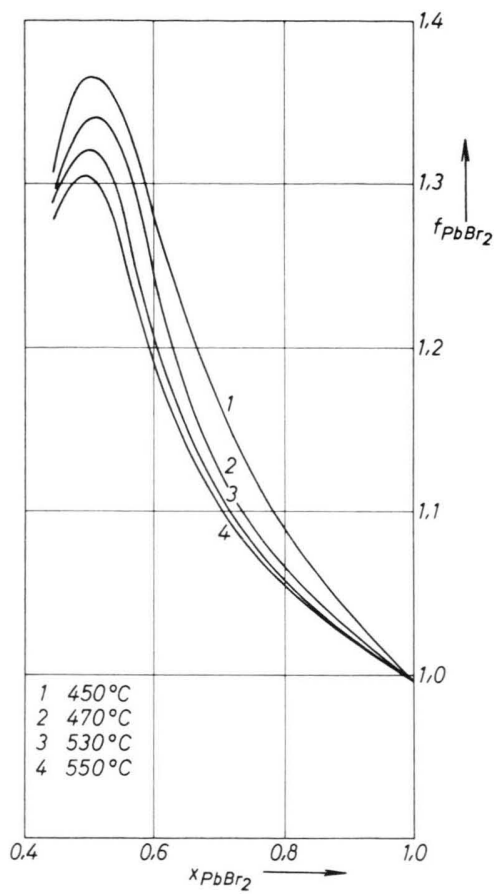

Abb. 2. Die Aktivitätskoeffizienten des Bleibromids in der Elektrolytschmelze $\mathrm{PbCl}_{2}+\mathrm{PbBr}_{2}$ bei verschiedenen Temperaturen in Abhängigkeit vom stöchiometrischen Molenbruch des Bleibromids.

Systeme mit drei Komponenten sind bisher noch sehr wenige untersucht worden. Aktivitätskoeffizienten für solche Systeme wurden, soweit uns bekannt ist, noch gar nicht berechnet. Es gibt einige Arbeiten mit Messungen an Konzentrationsketten mit Überführung, so die von Blander et al. ${ }^{15}$ und Hill et al. ${ }^{16}$. In jüngster Zeit erschien eine Arbeit mit

15 M. Blander, F. F. Blankenship u. R. F. Newton, J. Phys. Chem. 63, 1259 [1959].

16 D. G. Hill, J. Braunstein u. M. Blander, J. Phys. Chem. 64, 1038 [1960]. 
Messungen an einer chemischen Kette von Guion et al. ${ }^{17}$. Sie untersuchten das System $\mathrm{KCl}+\mathrm{AgCl}+\mathrm{NaCl}$ in Abhängigkeit von Temperatur und Zusammensetzung. Daraus lassen sich Aktivitätskoeffizienten berechnen, die ein interessantes Resultat liefern. Die von den Autoren untersuchte Kette enthält eine Silber- und eine $\mathrm{Cl}_{2}(\mathrm{C})$-Elektrode. Es lassen sich also primär die Aktivitätskoeffizienten des Silberchlorids in der Mischung bestimmen. Mit $q=-1$ und $v_{1}=v_{2}=v_{3}=2$ vereinfacht sich Gl. (53) auf die gewohnte Form

$$
\ln f_{2}=\frac{F\left(\Phi_{0}-\Phi\right)}{R T}-\ln x_{2} .
$$

Die Ergebnisse sind in Tab. 3 aufgeführt. In ihr bedeutet

$$
N=n_{\mathrm{NaCl}} /\left(n_{\mathrm{NaCl}}+n_{\mathrm{KCl}}\right),
$$

\begin{tabular}{cccccc}
\hline$x_{2}$ & & \multicolumn{5}{c}{$f_{2}$} \\
& $N=0,00$ & $N=0,25$ & $N=0,50$ & $N=0,75$ & $N=1,00$ \\
\hline 1,0 & 1,00 & 1,00 & 1,00 & 1,00 & 1,00 \\
0,5 & 0,84 & 0,91 & 0,97 & 1,06 & 1,10 \\
0,3 & 0,70 & 0,81 & 0,94 & 1,12 & 1,23 \\
0,2 & 0,62 & - & - & - & - \\
0,05 & 0,53 & 0,69 & 0,93 & 1,14 & 1,45 \\
\hline
\end{tabular}

Tab. 3. Aktivitätskoeffizienten für das System $\mathrm{KCl}+\mathrm{AgCl}+\mathrm{NaCl}$ bei $1073,2 \mathrm{~K}$.

wobei $n_{j}$ die Molmenge der Komponente $j$ ist. $N$ ist kein echter Molenbruch, da die dritte Komponente im Nenner der Gl. (69) fehlt. $N=0$ heißt, es ist kein $\mathrm{NaCl}$ im System. Die zweite Spalte gibt also die Aktivitätskoeffizienten für $\mathrm{AgCl}$ in der binären Elektrolytschmelze $\mathrm{KCl}+\mathrm{AgCl}$ wieder. Alle Angaben gelten für $800{ }^{\circ} \mathrm{C}=1073,2 \mathrm{~K}$.

Nach rechts nimmt der Gehalt an $\mathrm{NaCl}$ solange zu, bis kein $\mathrm{KCl}$ mehr in der Schmelze ist. Die Werte sind in Abb. 3 graphisch dargestellt. Man beobachtet, wie die negative Abweichung von der idealen Mischung, ausgehend vom System $\mathrm{KCl}+\mathrm{AgCl}$, sich umwandelt in eine positive Abweichung von der idealen Mischung bis zum binären System $\mathrm{NaCl}+\mathrm{AgCl}$. Die negative Abweichung von der idealen Elektrolytschmelze des binären Systems $\mathrm{KCl}+\mathrm{AgCl}$ findet man durch Untersuchungen von Murgulescu und STERnberg ${ }^{18}$ sowie von STERN ${ }^{19}$ an diesem System bestätigt. Für $\mathrm{NaCl}+\mathrm{AgCl}$ läßt sich die positive $\mathrm{Ab}$ weichung nachweisen mit der schon oben zitierten

17 J. Guion, M. Blander, D. Hengstenberg u. K. HageMARK, J. Phys. Chem. 72, 2086 [1968].

18 I. G. Murgulescu u. S. Sternberg, Rev. Chim. Acad. Rep. Populaire Roumaine 2, 251 [1957].

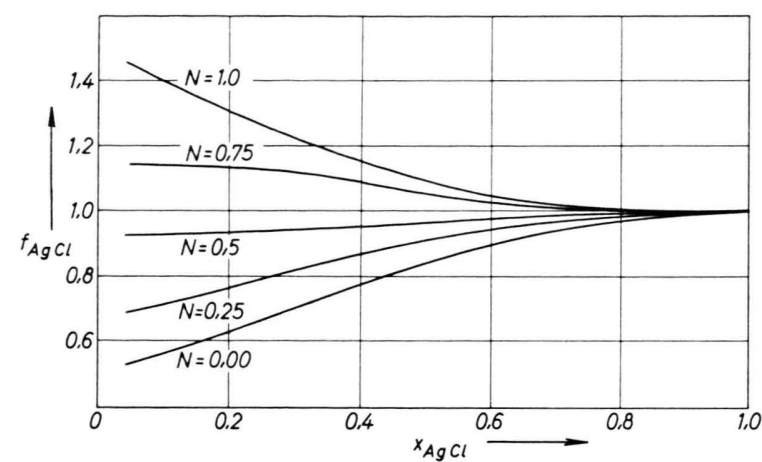

Abb. 3. Die Aktivitätskoeffizienten des Silberchlorids in der ternären Schmelze $\mathrm{KCl}+\mathrm{AgCl}+\mathrm{NaCl}$ bei $1073,2 \mathrm{~K}$ in $\mathrm{Ab}$ hängigkeit vom stöchiometrischen Molenbruch des Silberchlorids. $N$ ist das Verhältnis $n_{\mathrm{NaCl}} /\left(n_{\mathrm{NaCl}}+n_{\mathrm{KCl}}\right)$.

Arbeit von Panish et al. ${ }^{9}$, ferner durch frühere Untersuchungen von STERN ${ }^{20}$ sowie von STERNBERG et al. ${ }^{21}$. Zwischen $N=0,50$ und $N=0,75$ durchläuft $f_{\mathrm{AgCl}}$ in diesem System einmal die Gerade Eins. Dies bedeutet noch nicht, daß das System in dieser Zusammensetzung eine ideale Mischung darstellt. Die Voraussetzung dafür wäre, daß auch $f_{\mathrm{KCl}}$ und $f_{\mathrm{NaCl}}$ gleichzeitig Eins wären.

\section{Schlußbemerkungen}

Die angegebene Gl. (53) erlaubt, Aktivitätskoeffizienten aus EMK-Messungen für beliebig zusammengesetzte Elektrolytschmelzen zu bestimmen. Die Definition der Aktivitätskoeffizienten weicht von der bisher in der Literatur benutzten, die in Gl. (61) angegeben ist, ab. Bei der Auswertung stellt sich heraus, daß die beiden Definitionen für einige einfache Systeme übereinstimmen, bei denen alle Komponenten die gleiche Zerfallszahl haben und denen allen ein und dasselbe Ion gemeinsam ist, das auch in allen Komponenten die gleiche Zerfallszahl hat. Für das Ion der betrachteten Komponente $k$, das nicht in den anderen Komponenten enthalten ist, muß außerdem $v_{k \alpha}=1$ sein. Für alle anderen Systeme stimmen die Definitionen nicht überein. Unsere Definition hat den Vorteil, daß sich der Logarithmus der so definierten Aktivitätskoeffizienten für alle Systeme in Reihen entwickeln läßt, die für alle Grenzgesetze der betrachteten Elektrolytschmelzen von prinzipieller Bedeutung sind.

19 K. H. Stern, J. Phys. Chem. 60, 679 [1956].

20 K. H. Stern, J. Phys. Chem. 62, 385 [1958].

21 S. Sternberg u. S. Gheorghiu, Studii Cercet. Chim. Acad. Rep. Populaire Roumaine 7, 107 [1995]. 
Zum Schluß sei noch darauf hingewiesen, daß die von uns früher ${ }^{4}$ definierten Aktivitätskoeffizienten in Elektrolytschmelzen sich von den hier behandelten im wesentlichen durch die verschiedenen Konstanten unterscheiden, die auftreten, wenn man die Grenzübergänge $x_{j} \rightarrow 1$ durchführt. Für die Aktivitätskoeffizienten selbst, deren Reihenentwicklungen und für die daraus ableitbaren Grenzgesetze ist das bedeutungslos. Für die Zusatzfunktionen aber ist die von $\mathrm{HAASE}^{1}$ gewählte Normierung günstiger und von den Nichtelektrolytlösungen her gewohnter.
Damit keine zusätzliche Verwirrung auftritt, schlagen wir vor, die hier verallgemeinerten Aktivitätskoeffizienten zu verwenden, die für die reinen Komponenten in jedem System Eins werden. Es sei noch darauf hingewiesen, daß der in der vorliegenden Arbeit nicht benötigte Begriff der „Aktivität“ einer Komponente in einer Elektrolytschmelze von allen Autoren in gleicher Weise wie bei Nichtelektrolytlösungen verwendet wird ${ }^{1}$.

Herrn Prof. Dr. R. HAASE danke ich für sein Interesse und für die Unterstützung der Arbeit. 